\title{
BRIEF OVERVIEW OF THE WORLD HEALTH ORGANIZATION "VECTOR CONTROL GLOBAL RESPONSE 2017-2030" AND "VECTOR CONTROL ADVISORY GROUP” ACTIVITIES
}

\author{
R. VELAYUDHAN \\ Coordinator Vector Ecology and Management, Department of Control of Neglected \\ Tropical Diseases, World Health Organization, 20 Avenue Appia, CH-1211 Geneva \\ 27, Switzerland; VelayudhanR@who.int
}

\begin{abstract}
SUMMARY
More than $80 \%$ of the world's population is at risk of vector-borne disease and many of these diseases are concentrated in the poorest communities in tropical and subtropical regions; they cause unacceptable mortality and morbidity and impede economic growth. A new and comprehensive approach to preventing diseases and responding to outbreaks is clearly needed - one that engages multiple sectors and communities and is based on the best available evidence base. The 70th World Health Assembly adopted the strategic approach outlined in a new WHO Global Vector Control Response (GVCR) for 2017-2030. The response aims to reduce the burden and threat of vector-borne diseases through effective, locally adapted, and sustainable vector control. It is an approach for tackling multiple vectors and diseases that requires action across many sectors beyond health, including environment, urban planning, education, etc. The recent outbreaks of Aedes-borne diseases call for concerted action to deal with increased urbanization, erratic water supply, increased movement of people and commodities, altered land use patterns, and other environmental changes, including climatic changes that extend the distribution of vectors and pathogen transmission to more temperate climes. The GVCR provides a unique opportunity to work together and address the increasing burden of vector-borne diseases. This new approach is supported by the Vector Control Advisory Group (VCAG) that was established by WHO in 2013 in order to carry out independent evaluation of the public health value of innovative new tools, technologies and approaches for vector control and to enable WHO to provide evidence-based advice to Member States on whether their deployment and strategy is the most appropriate for their specific circumstances.
\end{abstract}

Key Words: Vector-borne diseases, public health, emerging diseases, inter-sectoral collaboration, mosquitoes, strategic framework, integrated vector management (IVM), health education, Aedes, dengue, malaria, chikungunya, yellow fever, Zika, WHO

633

J. Hendrichs, R. Pereira and M. J. B. Vreysen (eds.), Area-Wide Integrated Pest Management: Development and Field Application, pp. 633-644. CRC Press, Boca Raton, Florida, USA.

(C) 2021 IAEA 


\section{BACKGROUND}

Cognizant of recent major outbreaks of diseases such as dengue, malaria, chikungunya, yellow fever and Zika, as well as other emerging and persistently important diseases, Member States at the $139^{\text {th }}$ executive board meeting of the World Health Organization (WHO) requested the director-general in May 2016 to devise an appropriate response.

Development of the Global Vector Control Response 2017-2030 (GVCR) (WHO 2017) commenced immediately through a fast-tracked broad consultative process that was co-led by three departments within the Communicable Diseases cluster: the Global Malaria Programme (GMP), the Department of Control of Neglected Tropical Diseases (NTD), and the Special Programme for Research and Training in Tropical Diseases (TDR).

The strategic approach was welcomed one year later by Member States at the $70^{\text {th }}$ World Health Assembly, and a dedicated resolution was adopted (WHA 2017). The GVCR (WHO 2017) and associated resolution (WHA 2017) set out a new strategy to strengthen vector control worldwide through increased capacity, improved surveillance, better coordination and integrated action across diseases and sectors. It aims to reduce the burden and threat of vector-borne diseases through effective, locally adapted and sustainable vector control, and to prevent epidemics by 2030 in line with the United Nations Sustainable Development Goal 3 (Good Health and Well-being).

\section{RATIONALE}

- Major vector-borne diseases account for an estimated $17 \%$ of the global burden of all infectious diseases, and disproportionately affect human populations in poor countries.

- These diseases impede economic development through direct medical costs and indirect costs such as lost productivity and tourism.

- Social, demographic and environmental factors have caused increases in many vector-borne diseases in recent years, with major outbreaks of dengue, malaria, chikungunya, yellow fever and Zika since 2014.

- Most vector-borne diseases are preventable by various means, including vector control, if well implemented. Strong political commitment and improved investments have already led to major reductions in malaria, onchocerciasis and Chagas.

- The full impact of vector control against other diseases has yet to be achieved, but it is possible through re-alignment of programmes to optimise delivery of interventions that are tailored to a local context.

- This requires improved public health entomology capacity, a well-defined national research agenda, better coordination within and between sectors, strengthened monitoring systems, community involvement, and availability and use of more interventions with proven public health value. 


\section{NEED FOR A GLOBAL VECTOR CONTROL RESPONSE}

Never has the need for a comprehensive approach to vector control to counter the impact of vector-borne diseases been more urgent. The unprecedented global spread of dengue and chikungunya viruses, as well as outbreaks of Zika and yellow fever in 2015-2016, clearly highlight the challenges faced by numerous countries. Transmission and risk of vector-borne diseases are rapidly changing due to unplanned urbanization, increased movement of people and goods, environmental changes and biological challenges, such as vectors populations becoming resistant to insecticides and evolving strains of pathogens. In particular, rapid, unplanned urbanization in tropical and sub-tropical areas renders large human populations at elevated risk of emergence and expansion of arboviral diseases spread by mosquitoes. Many countries are still unprepared to address these looming challenges (the current global distribution of seven major vector-borne diseases is shown in Fig. 1).

The strong influence of social and environmental factors on vector-borne pathogen transmission underscores the critical importance of flexible vector control delivery and monitoring, as well as evaluation systems that support locally tailored approaches. Re-alignment of national programmes to optimize implementation of interventions against multiple vectors and diseases will maximize the impact of available resources. Health systems must be prepared to detect and respond quickly and effectively to changes. This rapid response requires not only the availability of effective, evidence-based control interventions, but also well-trained staff who can build sustainable systems for their area-wide delivery. To achieve these goals, reforms to vector control programmatic structures are urgently needed.

Vector-borne diseases are everyone's problem, not just the health sector. Achievement of the United Nations Sustainable Development Goal 3 to ensure good health and well-being will rely on effective vector control, as will initiatives for clean water and sanitation (Goal 6), sustainable cities and communities (Goal 11) and climate action (Goal 13). Multiple approaches that are implemented by different sectors will be required for control and elimination of vector-borne disease, such as those promoting healthy environments (Pruss-Ustun et al. 2016). Engaging local authorities and communities as part of broad-based inter-sectoral collaboration will be key to improved vector control delivery, through tailoring of interventions to specific scenarios as informed by local entomological, epidemiological and ecological data. Building sustainable control programmes that are resilient in the face of technical, operational, climatic and financial challenges will require the engagement and collaboration of local communities.

Recent advances to modernize and develop new vector control and surveillance tools means that there has never been a better time to reinvigorate vector control. To be effective, strong political commitment and long-term investment are needed. The GVCR response seeks neither to replace or override existing disease-specific strategies nor to shift the focus from other essential interventions, such as vaccines against yellow fever, Japanese encephalitis and tick-borne encephalitis, massadministration of medicines against lymphatic filariasis and human onchocerciasis, and artemisinin-based combination therapy against malaria. Rather, it aims to add to these efforts and help countries mount coherent and coordinated efforts to address the increasing burden and threat of vector-borne diseases. 


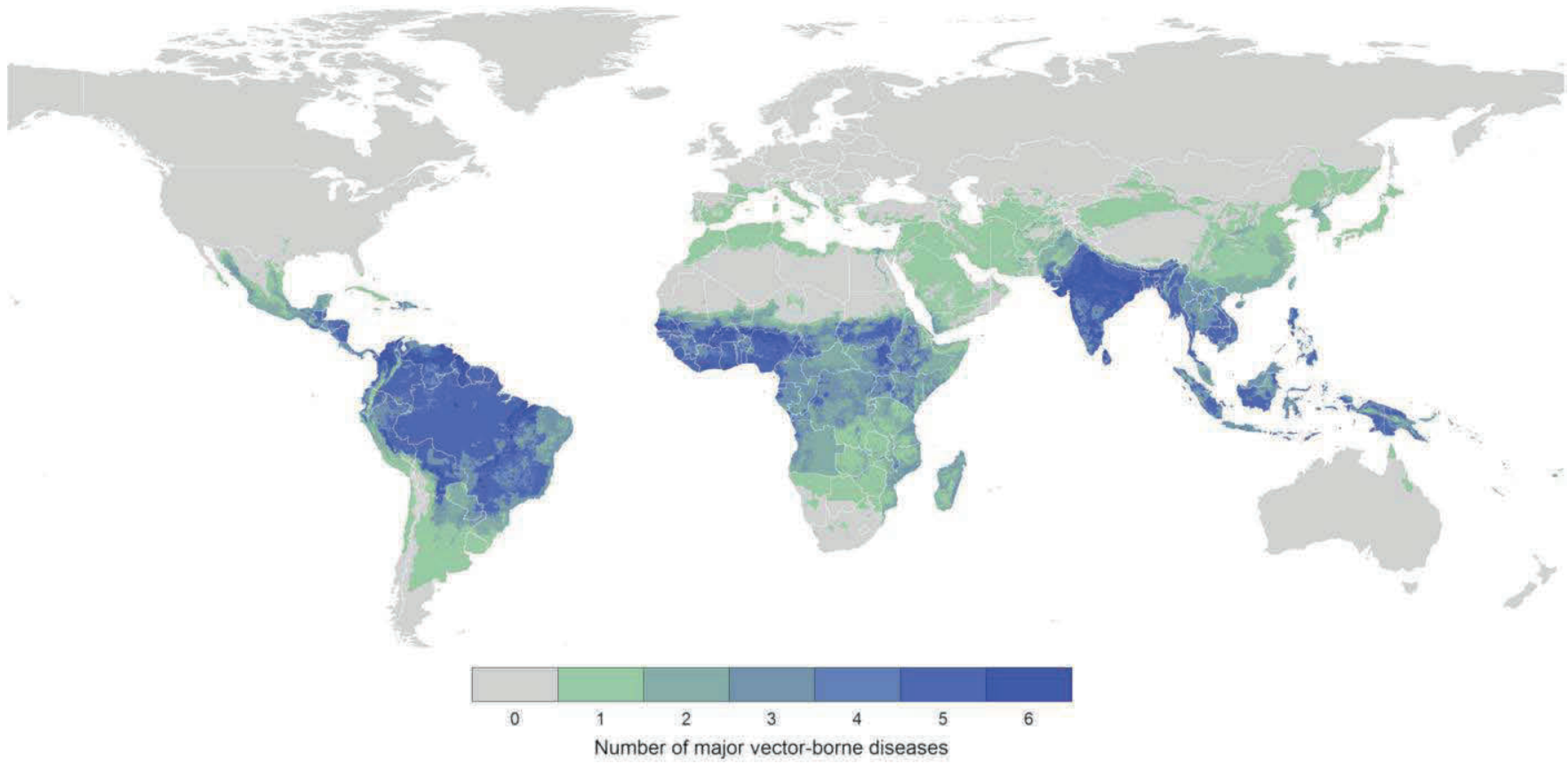

Figure 1. Combined global distribution of seven major vector-borne diseases for which integration of vector control programmes may be beneficial: malaria, lymphatic filariasis, leishmaniasis, dengue, Japanese encephalitis, yellow fever and Chagas disease transmission in 2015. Colours indicate the number of vector-borne diseases that pose a risk at each $5 \times 5 \mathrm{~km}$ grid cell (from Golding et al. 2015). 
The GVCR provides strategic guidance to countries and development partners to urgently strengthen vector control as a fundamental approach to preventing diseases and responding to outbreaks. To achieve this, re-alignment of vector control programmes is required, supported by increased technical capacity, strengthened monitoring and surveillance systems, and improved infrastructure. Ultimately, this will support implementation of a comprehensive approach to vector control that will enable the achievement of disease-specific national and global goals and contribute towards achievement of the United Nations Sustainable Development Goals.

\section{THE GLOBAL VECTOR CONTROL RESPONSE AT A GLANCE}

\subsection{Vision}

A world free of human suffering from vector-borne diseases.

\section{2. $\operatorname{Aim}$}

To reduce the burden and threat of vector-borne diseases through effective locallyadapted and sustainable vector control.

\subsection{Goals for 2020-2030}

The goals, including 2020-2030 milestones and targets, are presented in Table 1.

Table 1. Goals for 2020-2030 on the global vector control response

\begin{tabular}{|l|l|l|l|}
\hline \multicolumn{1}{|c|}{ Milestones } & \multicolumn{2}{|c|}{ Targets } \\
\cline { 2 - 4 } & \multicolumn{1}{|c|}{2020} & \multicolumn{1}{c|}{2025} & \\
\cline { 2 - 4 } $\begin{array}{l}\text { Reduce mortality due to } \\
\text { vector-borne diseases } \\
\text { globally compared to } \\
2016\end{array}$ & At least 30\% & At least 50\% & At least 75\% \\
\hline $\begin{array}{l}\text { Reduce case incidence } \\
\text { due to vector-borne } \\
\text { diseases globally } \\
\text { compared to 2016 }\end{array}$ & At least 25\% & At least 40\% & At least 60\% \\
\hline $\begin{array}{l}\text { Prevent epidemics of } \\
\text { vector-borne diseases }\end{array}$ & & $\begin{array}{l}\text { In all countries } \\
\text { without } \\
\text { transmission in } \\
2016\end{array}$ & $\begin{array}{l}\text { In all } \\
\text { countries }\end{array}$ \\
\hline
\end{tabular}

${ }^{*}$ Rapid detection of outbreaks and curtailment before spread beyond country or region 


\subsection{Priority Activities for 2017-2022}

For the period 2017-2022 the priority activities are as follows (they will be revised and updated for the subsequent period of 2023-2030):

1. National and regional vector control strategic plans developed and adapted to align with the global vector control response.

2. An assessment of national vector control needs carried out or updated, and a resource mobilization plan developed (including for outbreak response).

3. National entomology and cross-sectoral workforce appraised and enhanced to meet identified requirements for vector control, including for epidemic response and pre-emptive response.

4. Relevant staff from Ministries of Health and/or their supporting institutions trained in public health entomology and sustainable career pathway is established.

5. National and regional institutional networks to share data and support training and education in public health entomology, and technical support established and functioning.

6. National agendas for basic and applied research on entomology and vector control established and/or progress reviewed.

7. National inter-ministerial task forces and steering committee for multi-sectoral engagement in vector control established and functioning.

8. National vector surveillance systems strengthened and integrated with health information systems to guide vector control. Cross border exchange of information is encouraged.

9. National plans for effective community engagement and mobilization in vector control developed.

10. National targets for protection of at-risk populations with appropriate vector control aligned across vector-borne diseases.

\section{BEYOND INTEGRATED VECTOR MANAGEMENT}

Integrated vector management (IVM) is a rational decision-making process for the optimal use of resources for vector control, as presented in a WHO global strategic framework released in 2004, a WHO position statement issued in 2008, and other supporting documents (WHO 2004, 2008, 2016).

While this approach seeks to improve the efficacy, cost-effectiveness, ecological soundness and sustainability of disease-vector control, uptake has been poor, due to insufficient political buy-in for reorientation of programmes to support a harmonized approach to vector control across diseases. This has largely been due to limited human capacity to advocate, plan and implement, as well as fragmented global and national architecture to support a multi-disease approach.

Given the recent alarming increase in numerous vector-borne diseases and the serious threat posed to economic development, the GVCR response aims to reposition vector control as a key approach to prevent, manage, or eliminate vectorborne diseases. It builds on the basic concept of IVM with renewed focus on improved human capacity at national and sub-national levels, as well as strengthened infrastructure and systems, particularly in areas vulnerable to vector-borne disease 
upsurges (e.g. sustainable development, access to potable water, waste management, home construction, community design, water supply and solid waste disposal).

For sustainable impact in vector control, increased inter-sectorial and interdisciplinary action is essential, linking efforts in environmental management, health education, and reorienting relevant government programmes around proactive strategies that will improve living conditions and control new and emerging threats. Critical attention is given in the GVCR to current opportunities available for leverage, as well as challenges that need to be addressed in order to enable effective and sustainable vector control with interventions that will work best for the specific/unique circumstances of each local situation.

\section{OPPORTUNITIES FOR GLOBAL VECTOR CONTROL RESPONSE}

Many opportunities exist to enhance the impact of vector control. The overall benefit of enhancing capacity and capability is a critical opportunity that is essential for a successful GVCR, will have a positive impact across diseases, and is essential for sustainability:

1. Development. Environmentally sustainable and resilient development in urban centres (Habitat III 2016; UNECE 2014-2015) that reduces poverty and improves living standards will reduce transmission of vector-borne pathogens.

2. Recognition. Existing global and regional strategies against vector-borne diseases demonstrate their importance in the global health agenda and in other sectors, and represent high-level commitment for their reduction, elimination and, for some, eradication.

3. Expansion. Recent successes in vector control, such as against vectors of malaria, onchocerciasis and lymphatic filariasis, have led to major reductions in vector-borne diseases. Further impacts could be achieved through sustained and expanded use of proven vector control interventions.

4. Optimization. Re-aligning national programmes to optimize implementation of vector control against multiple vectors and diseases, across geographic areas and human populations, will leverage available resources to maximum impact of IVM (Fig. 2).

5. Collaboration. Building on existing collaborations across ministries, sectors, partners and networks to share data and expertise will improve timely access to information and resources for the most effective vector-borne disease control. Regional and cross-border collaboration further helps in tracking outbreaks and responding in a timely manner.

6. Adaptation. The strong influence of social, demographic and environmental factors on vector-borne pathogen transmission underscores the critical importance of flexible vector control delivery, monitoring, and evaluation systems that support locally-tailored approaches that can be adapted to specific opportunities or challenges.

7. Innovation. Development of novel tools, technologies and approaches such as new insecticides and formulations, vector traps and baits, use of Wolbachia spp. and genetic modification for population reduction or replacement, other forms of vector sterilisation, larviciding via auto-dissemination, endectocides, spatial repellents and 
vapor active insecticides, and housing improvements to exclude vectors and reduce favourable harbourages have the potential to further reduce disease burden.

8. Technology. Advances that support evidence-based vector control such as information communication technologies that support real-time data capture or social media, or risk stratification and predictive geo-informatics tools such as geographic information systems, remote sensing, and climatic models can be leveraged to further optimize planning and implementation.

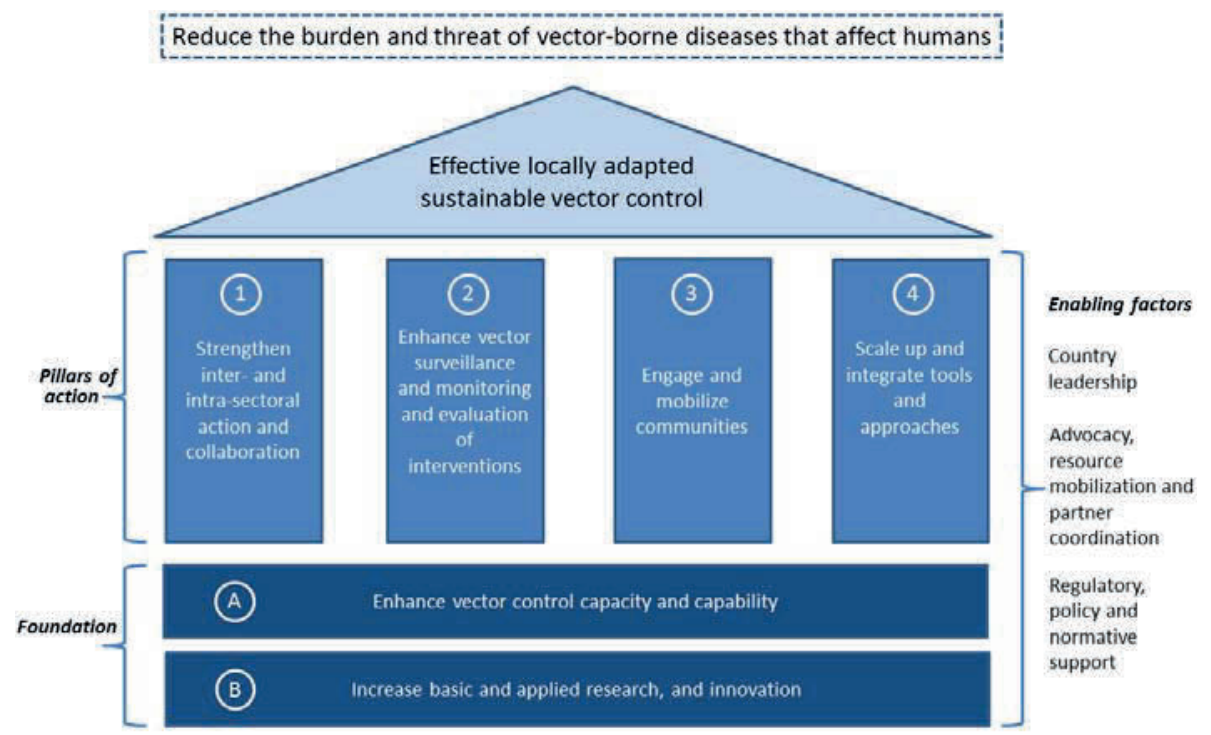

Figure 2. Response framework from the Global Vector Control Response (WHO 2017).

\section{CHALLENGES OF GLOBAL VECTOR CONTROL RESPONSE}

While there are many opportunities to enhance the impact of vector control, there are also multiple interconnected challenges that impede progress against vector-borne diseases and that need to be overcome. Threats to effective and impactful vector control can be grouped as systemic, structural, informational, environmental, human movement, and political and financial.

1. Systemic. Capacity for vector surveillance and control is insufficient in most countries at risk from vector-borne diseases. With a few notable exceptions, vectorborne disease prevention programmes at national and subnational levels have limited public health entomology capacity and poor infrastructure. Career structures for technical specialists and technicians within the health system are absent or weak. Establishing basic capacity provides enormous impact across multiple vector-borne diseases and sectors.

2. Structural. Many countries that are endemic for more than one major vectorborne disease have disease-specific programmes and strategies that do not optimally 
leverage synergies and sometimes compete for resources. Well-funded programmes - such as for malaria in some countries of sub-Saharan Africa - are often expected to respond to outbreaks of other vector-borne diseases without adequate capacity and additional resources at the expense of routine, core activities.

3. Informational. The evidence base to support effective vector control is limited for most vector-borne diseases due to lack of research support. Vector surveillance remains weak in many countries despite insecticide resistance and changes in vector behaviour threatening the efficacy of current interventions. Available information is not used to its full potential to guide control interventions.

4. Environmental. Changes in vector habitats such as those due to rapid urbanization or alterations in land use, water management and farming practices are often unpredictable, uncontrollable, and complex. Climatic changes that extend the distribution of vectors and pathogen transmission to more temperate climes are also of concern. With two thirds of the global population expected to live in urban settings by 2050 (United Nations 2014), large populations in tropical and subtropical zones will be at increasing risk of Aedes-borne diseases.

5. Movement of humans and goods. Increased global human population movement due to changing travel patterns, migration for employment, or displacement resulting from humanitarian crises, as well as increased global trade are likely to accelerate the introduction of invasive species or pathogens into new receptive areas and expose non-immune populations to novel infections and disease.

6. Political and financial. Substantial financial support has been provided to scale up of the use of insecticide-treated bed nets and indoor residual spraying against malaria vectors since 2000. However, there has been insufficient focus on scale-up and effective delivery of vector control for other vector-borne diseases, especially in the absence of either large epidemics or high mortality rates.

\section{THE GLOBAL VECTOR CONTROL RESPONSE IN SUMMARY}

The GVCR aims to reduce the burden and threat of vector-borne diseases through effective locally-adapted, sustainable, and inter-sectorial vector control. Success will depend on the ability of countries to overcome current limitations and to strengthen the capacity, financial resources and inter-sectorial collaboration of their vector surveillance and control programmes. The WHO will support the development of regional action plans and country activities based on GVCR strategies and priorities identified in the Country Cooperation Strategy (CCS) that guides WHO work in each country to support its national health strategy.

The key areas of activity that intend to radically change the control of vectorborne diseases are:

- Aligning action across sectors, since vector control is more than the work done by the Ministry of Health $(\mathrm{MOH})$. The $\mathrm{MOH}$ has to coordinate work with other relevant ministries and with city planners to reduce or build out habitat of urban vectors (Aedes mosquitoes, rodents, etc.).

- Engaging and mobilizing communities to become active partners in the GVCR, which will protect themselves and build resilience against future disease outbreaks (including the impact of climate change). 
- Enhancing surveillance to trigger early responses to increased risk in disease or vector populations, and to identify when and why interventions are not working as expected.

- Encouraging pre-emptive programmes that use surveillance data to reduce or eliminate the vectors wherever feasible, and

- Scaling-up vector control tools (including the integration of new tools and innovative approaches) and using them in combination to maximize impact on disease, while minimizing impact on the environment.

Specifically, the new integrated approach calls for national programmes to be realigned so that public health workers can focus on the complete spectrum of relevant vectors and thereby control all the diseases they cause. Recognizing that efforts must be adapted to local needs and sustained, the success of the response will depend on the ability of countries to strengthen their vector control programmes and enhance their inter-sectorial collaborations, including with sectors that are not currently part of "traditional" vector control. National strategic plans need to be revised and country-specific targets defined till 2030. All the relevant GVCR documents and related links can be downloaded from WHO (2017).

\section{VECTOR CONTROL ADVISORY GROUP}

In 2013 the WHO established a Vector Control Advisory Group (VCAG) to assist $\mathrm{WHO}$ in assessing innovative approaches, new tools, and technologies for vector control, and to serve as an advisory body to WHO on new approaches of vector control for malaria and other vector-borne diseases. During the very early stages of innovation, scientists and developers of such new products or approaches can bring new ideas or new intervention concepts for feedback to the VCAG.

If the product developers wish, VCAG can provide advice on the type and depth of evidence that will likely be used for the assessment, providing an opportunity for product developers to align with VCAG on overall evidence requirements before the launch of resource-intensive activities such as large-scale epidemiological trials (randomized control trials with epidemiological end-point). The advice will be provided in individual discussions between the product developers and the group at the VCAG meeting. It may cover, for instance, the needs concerning evidence of epidemiological and vector control outcomes, epidemiological mode of action, economic feasibility or user acceptability. To support its deliberations, VCAG may consider the initial results of tests and studies carried out by the product developers.

The VCAG is jointly managed by the Global Malaria Programme (GMP), the Department of Control of Neglected Tropical Diseases (NTD), and the WHO Prequalification Team for the assessment of vector control products (VCAG 2019).

In summary the VCAG objectives are:

- To assess the public health value of new vector control tools, technologies and approaches submitted to WHO for evaluation.

- To provide guidance to product developers on data requirements and study designs to generate the evidence required for a VCAG assessment. 
- To provide guidance to WHO and its policy advisory groups, the MPAC (Malaria Policy Advisory Committee) and the STAG (Strategic and Technical Advisory Group for neglected tropical diseases), on the public health value of new tools, technologies and approaches.

Once a relevant body of evidence has been presented to VCAG, which contains an indication of the epidemiological outcome of the new form of vector control, VCAG will review all available scientific evidence (which may also include available sources other than the data presented by the product developers). Based on this review, VCAG evaluates the public health value of the new intervention by answering questions such as: "Is this new intervention efficacious for some defined public health purpose (in disease prevention through vector control) and in some defined circumstances, and will it be useful to and feasible for its intended users?" The answer might in some instances requires additional evidence.

The new approaches or products ongoing assessment or that are in planning and are currently under consideration by VGAC are shown in Fig. 3.

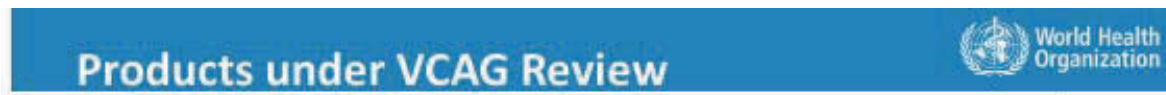

\begin{tabular}{|c|c|c|}
\hline New Product - Variation & Disease Target & Step 3 \\
\hline ITN against IR Vector (extend ITN) & Malaria & ongoing \\
\hline Treated walls against IR vector (extend IRS) & Malaria & N.A. \\
\hline Peri-focal residual spraying (extend IRS) & $\begin{array}{l}\text { Dengue and Aedes-borne arboviral diseases, } \\
\text { Leishmaniasis }\end{array}$ & planning \\
\hline Insecticide-treated curtain (extend ITN) & Malaria & N.A. \\
\hline New Product Class - (chemical) & Generic Exemplar & \\
\hline Attract and kill baits & Malaria & ongoing \\
\hline Spatial repellents & Malaria, Dengue and Aedes-borne arboviral diseases & ongoing \\
\hline ITM for specific risk groups & Malaria & N.A. \\
\hline Vector traps & Dengue and Aedes-borne arboviral diseases & ongoing \\
\hline Lethal house lures & Malaria & ongoing \\
\hline Systemic insecticide & Leishmaniasis & planning \\
\hline New Product Class - (biological) & Generic Exemplar & \\
\hline Microbial control in adult vectors & Dengue and Aedes-borne arboviral diseases & ongoing \\
\hline Pop. reduction through genetic manipulation & Dengue and Aedes-borne arboviral diseases (self limiting) & planning \\
\hline Population reduction & Malaria (gene-drive) & N.A. \\
\hline Pop. alteration of malaria vector mosquitoes & Malaria (gene-drive) & N.A. \\
\hline SIT \& incompatible insect technique (IIT) & Dengue and Aedes-borne arboviral diseases & planning \\
\hline
\end{tabular}

Figure 3. New products or innovative approaches under consideration by the Vector Control Advisory Group (VCAG) of WHO. Further details on the VCAG can be found under VGAG (2019). IIT = Incompatible Insect Technique using Wolbachia; IR = insecticide resistant;

$I R S=$ indoor residual spraying; ITM = insecticide-treated material; ITN = insecticidetreated nets; N.A. =not applicable at present since it is yet to complete preliminary studies; SIT $=$ Sterile Insect Technique. Step 3 refers to Phase 3 studies for assessing impact on the disease/infection or both. 
As soon as the VCAG decides that the answer to this question is "yes", and that proof-of-principle has indeed been established for the new form of vector control, responsibility within WHO for further review will pass to the advisory bodies MPAC and STAG for the WHO technical department(s) (e.g. Departments of Control of Neglected Tropical Diseases and Global Malaria Programme) responsible for the particular vector-borne disease(s) against which the new intervention is considered likely to be useful.

Hence, after validating the value of the new form of vector control, VCAG will present its results to WHO for consideration and submission to MPAC and/or STAG in their respective meetings, expressing its opinion on the usefulness of the new intervention.

\section{REFERENCES}

Golding, N., A. L. Wilson, C. L. Moyes, J. Cano, D. M. Pigott, R. Velayudhan, S. J. Brooker, D. L. Smith, S. I. Hay, and S. W. Lindsay. 2015. Integrating vector control across diseases. BMC Medicine 13: 249.

Habitat III. 2016. Habitat III new urban agenda. United Nations Conference on Housing and Sustainable Urban Development. Final draft: 10 September 2016.

Pruss-Ustun, A., J. Wolf, C. Corvalan, R. Bos, and M. Neira. 2016. Preventing disease through health environments: A global assessment of the burden of disease from environmental risks. World Health Organization. Geneva, Switzerland.

UNECE (United Nations Economic Commission for Europe). 2014-2015. United smart cities: Smart urban solutions for transition and developing countries. United Nations Economic Commission for Europe, United Nations Economic and Social Council. Geneva, Switzerland.

United Nations. 2014. World urbanization prospects: The 2014 revision, highlights (ST/ESA/SER.A/352). United Nations, Department of Economic and Social Affairs, Population Division. New York, USA.

(VCAG) Vector Control Advisory Group. 2019. Global Malaria Programme, the Department of Control of Neglected Tropical Diseases, and the WHO Prequalification Team for Vector Control Products. World Health Organization. Geneva, Switzerland.

(WHA) World Health Assembly. 2017. Global vector control response: An integrated approach for the control of vector-borne diseases. Agenda item 14.2 31 May 2017. Seventieth World Health Assembly, Resolution WHA70.16.

(WHO) World Health Organization. 2004. Global strategic framework for Integrated Vector Management. World Health Organization. Geneva, Switzerland.

(WHO) World Health Organization. 2008. WHO position statement on Integrated Vector Management. World Health Organization. Geneva, Switzerland.

(WHO) World Health Organization. 2016. A toolkit for integrated vector management in sub-Saharan Africa. World Health Organization. Geneva, Switzerland.

(WHO) World Health Organization. 2017. Global vector control response 2017-2030. World Health Organization. Geneva, Switzerland. 53 pp. 